\title{
Análisis de la producción científica española en investigación aeronáutica con un enfoque de género (1990-2005): un estudio de caso
}

Spanish scientific production on aerospace research from a genre perspective (1990-2005): a case study

Silvia Paz Otero (1), Rocío Herrerías Rubí (2) y Fernando Mérida Martín (3)

(1) Instituto Nacional de Técnica Aeroespacial (INTA), Carretera de Ajalvir, Km. 4, 28850 Torrejón de Ardoz (Madrid), pazos@inta.es (2) herreriasrr@inta.es (3) meridamf@inta.es

\section{Resumen}

Este artículo gira en torno a la situación de la mujer en el ámbito de la investigación aeronáutica y aeroespacial en el marco de la institución española de referencia en este sector, el Instituto Nacional de Técnica Aeroespacial(INTA). Con un enfoque de género se analizan los datos extraídos de un análisis bibliométrico previo referente al mencionado centro y al ámbito temporal acotado (1990-2005), datos obtenidos a través de sus publicaciones en el Web of Knowledge. Su actividad científica es valorada a través de los indicadores de productividad y autoría; áreas temáticas de interés prioritario para las mujeres, etc., todo ello encuadrado en el marco de una institución puntera en la investigación aeronáutica en el área española. Con los datos obtenidos se pretende poner de manifiesto la cada vez mayor incorporación de las mujeres no sólo al ámbito laboral de las ingenierías y otras carreras técnicas vinculadas a ellas, sino también a la investigación en estas áreas.

Palabras clave: Instituto Nacional de Técnica Aeroespacial. Bibliometría. Análisis de dominio. Estudios de género. Científicas.

\section{Introducción}

Hasta fechas relativamente recientes, era poco frecuente encontrar mujeres en las universidades y facultades españolas. Entre otros factores, la llegada de la Democracia a España supuso un cambio en esta realidad, al facilitar el acceso y la definitiva incorporación de las mujeres al ámbito universitario. Desde entonces, las mujeres se han convertido en el colectivo con mayor presencia en las universidades españolas, registrándose en este momento un mayor número de mujeres licenciadas que varones que concluyen sus estudios universitarios $(60,5 \%$ en el último año), según los últimos datos facilitados por el Ministerio de Educación y Ciencia. Al hilo de estos informes, es tendencia generalizada pensar que las mujeres españolas se decantan en

\begin{abstract}
This paper analyses the situation of women in the resarch activity within the aerospace sector. In particular, it takes into account their role in the main Spanish establishment for aeronautics and space research, The National Institute of Aeroespace Technique (INTA). From data obtained from a previous bibliometric study done at INTA for the period 19902005, a genre perspective analysis has been conducted. These data were collected from their publications in the Web Of Knowledge. Their scientific activity is evaluated through two indicators, productivity and authorship, temathic areas of specific interest for women, etc. With the data obtained, the increasing presence of women is shown, not only in engineering or other disciplines related with it, but also in research.
\end{abstract}

Keywords: National Institute of Aerospace Technique. Bibliometrics. Domain Analysis. Genre Studies. Women in Science.

mayor número por los estudios vinculados a las Ciencias Sociales y a las Humanidades.

No obstante, y cada vez en mayor grado, las mujeres se han incorporado decisivamente a los estudios técnicos, aumentando tanto su presencia como su relevancia, al incorporarse de manera efectiva a la investigación en estas áreas. De hecho, y según datos difundidos por la Oficina Estadística Comunitaria (Eurostat) en un Informe publicado en septiembre del año 2006, el $38,8 \%$ de los científicos e ingenieros en España son mujeres, un porcentaje superior a la media de la Unión Europea que se sitúa en el $29 \%$. El porcentaje sigue siendo bajo, pero considerablemente superior al registrado en otras épocas. Nuestro planteamiento es trasladar esta realidad a nuestro centro de trabajo, un 
Organismo Público de Investigación, el Instituto Nacional de Técnica Aeroespacial (INTA).

El INTA es el organismo público de referencia en la investigación aeronáutica y espacial desde su creación en 1942. A lo largo de más de sesenta años de historia ha realizado multitud de actividades en el ámbito de la investigación básica, aplicada y de desarrollo. Resultado de ese proceso investigador es su muy notable producción científica que se ha traducido, desde su creación, en la publicación de artículos en revistas científicas y técnicas, así como de monografías; en la colaboración en congresos y jornadas especializadas y también en la presentación de documentos adscritos a la denominada literatura gris como patentes, o normas. En particular, el INTA ha producido una notable cantidad de informes técnicos, memorandums y otro tipo de documentos resultantes de contratos con entidades públicas y privadas.

En la última edición de lbersid celebrada en Zaragoza en el año 2006, fueron presentados los datos de valoración global referidos al conjunto de la producción científica emitida por el INTA. En esta ocasión, pretendemos aportar desde una perspectiva de género, los datos relativos a la producción científica generada exclusivamente por las mujeres que desarrollan su vida profesional en nuestro centro de trabajo.

\section{Metodología}

El presente análisis surge de la inquietud por conocer la producción científico-técnica específicamente desarrollada por las mujeres del INTA dentro del cómputo global de la productividad del instituto. El INTA está integrado por varias dependencias: el campus de Torrejón de Ardoz, donde se ubica la mayor parte del Instituto; el CEDEA, ubicado en El Arenosillo, Huelva; y las estaciones espaciales de Villafranca del Castillo y de Maspalomas. Este estudio se limita sólo al INTA como nodo central.

Para realizar este estudio nos hemos basado en la base de datos del Institute for Scientific Information (ISI). El período analizado comprende los años 1990-2005, para el que se han procesado un total de 207 referencias dentro de las bases de datos $\mathrm{SCl}$ (Science Citation Index), IC (Index Chemicus), Current Chemical Reactions (CCR-Expanded). Estas referencias se han obtenido a través de las búsquedas en el campo "AD (ADRESS)", tratando de independizar los registros de las tres entidades de referencia (INTA, LAEFF, CAB).

El proceso de aislamiento de los datos no ha estado exento de complicaciones, puesto que no existe uniformidad en la adscripción de los autores a la organización de la que dependen. Tampoco ha resultado fácil cribar las referencias correspondientes a las mujeres. En este sentido, podemos afirmar que las bases de datos del ISI, recurso comúnmente utilizado para los análisis bibliométricos de producción científica, identifican a los autores y las referencias citadas sólo por sus apellidos y por las iniciales de su nombre, una identificación que resulta insuficiente para aislar los datos. De hecho, a nivel particular hemos tenido problemas para deshacer la ambigüedad en los nombres de algunos autores (por ejemplo, M. Rodríguez o A. Jiménez) cuyas iniciales y apellidos por ser excesivamente comunes no se podían identificar con total garantía de éxito.

\section{Resultados obtenidos}

\subsection{Productividad}

De las 207 referencias obtenidas, 131 corresponden a artículos y 76 a ponencias o comunicaciones remitidas a congresos. La tasa de productividad global anual de los autores del INTA es de 13 contribuciones (Tabla 1).

\begin{tabular}{lrrr}
\hline Año & Hombres & Mujeres & Total \\
\hline 1990 & 2 & 1 & 3 \\
1991 & 0 & 0 & 0 \\
1992 & 1 & 1 & 2 \\
1993 & 1 & 1 & 2 \\
1994 & 6 & 1 & 7 \\
1995 & 5 & 2 & 7 \\
1996 & 1 & 1 & 2 \\
1997 & 3 & 2 & 5 \\
1998 & 5 & 1 & 6 \\
1999 & 12 & 5 & 17 \\
2000 & 6 & 5 & 11 \\
2001 & 5 & 8 & 13 \\
2002 & 9 & 7 & 16 \\
2003 & 8 & 5 & 13 \\
2004 & 9 & 7 & 16 \\
2005 & 11 & 0 & 11 \\
TOTAL & 84 & 47 & 131 \\
\hline
\end{tabular}

Tabla I. Número de artículos por género.

El porcentaje medio de artículos firmados por hombres es de 5 contribuciones por año; el de mujeres, 3, pudiéndose establecer la media global en 8 artículos al año. El año más productivo es 1999, con 17 contribuciones (Tabla 2). Por sexos, 1999 es el año más productivo para los investigadores del INTA (12 contribuciones), 
mientras que el año 2001 lo es para las investigadoras (8 contribuciones).

\begin{tabular}{lrrr}
\hline Año & Hombres & Mujeres & Total \\
\hline 1990 & 0 & 0 & 0 \\
1991 & 0 & 0 & 0 \\
1992 & 0 & 0 & 0 \\
1993 & 1 & 1 & 2 \\
1994 & 2 & 0 & 2 \\
1995 & 2 & 1 & 3 \\
1996 & 4 & 0 & 4 \\
1997 & 2 & 2 & 4 \\
1998 & 4 & 4 & 8 \\
1999 & 1 & 6 & 7 \\
2000 & 6 & 8 & 14 \\
2001 & 2 & 3 & 5 \\
2002 & 8 & 3 & 11 \\
2003 & 5 & 5 & 10 \\
2004 & 4 & 2 & 6 \\
2005 & 0 & 0 & 0 \\
TOTAL & 41 & 35 & 76 \\
\hline
\end{tabular}

Tabla II. Número de relatorios a congresos porgénero.

En lo que se refiere a relatorios a congresos, seminarios, jornadas, etc., el porcentaje medio de contribuciones es de 5 , siendo 2 el valor medio establecido para las contribuciones que aparecen firmadas tanto por hombres como por mujeres. Esto demuestra una enorme similitud en los datos. El año más productivo es el año 2000 , con 14 contribuciones. Por sexos, tanto para un caso como para el otro, 8 es el número máximo de contribuciones, siendo 2002 el año más productivo para los investigadores del INTA, mientras que el año 2000 lo es para las investigadoras (Figura 1).

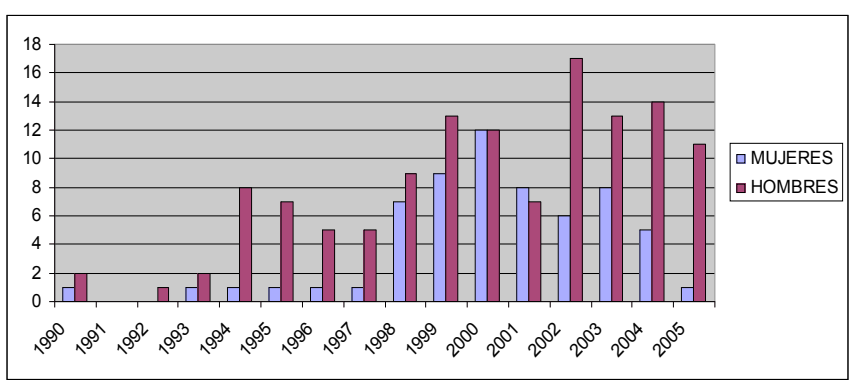

Figura 1. Distribución por años y por género de las contribuciones de los autores del INTA.

Tal y como se aprecia en el gráfico precedente, el año 2000 es el año en el que se registra un mayor número de contribuciones firmadas por las mujeres, mientras que el año 2002 es el que registramos valores máximos para los hombres. Es destacable el vacío que se registra en el binomio 1991-1992, en el que no contabilizamos en las bases del WOK ninguna contribución firmada por autores del INTA.

\subsection{Autoría.}

En cuanto al número de autores, fue posible determinar el género de los autores en un $97 \%$. Sólo en 2 casos fue imposible determinar el nombre completo de los autores, y, por lo tanto, su sexo (Tabla 3 ).

\begin{tabular}{lc}
\hline Sexo & Número de autores \\
\hline Hombres & 55 \\
Mujeres & 21 \\
No identificados & 2 \\
Total INTA & 78 \\
\hline
\end{tabular}

Tabla III. Número de autores por sexo.

La media española de personal femenino empleadas en actividades de I+D oscila en porcentajes en torno al $30 \%$, cifra que se eleva al $87 \%$ si nos referimos al mismo tipo de personal empleado en el INTA. De ese porcentaje registrado para el INTA, al menos un $30 \%$ son investigadoras (Figura 2).
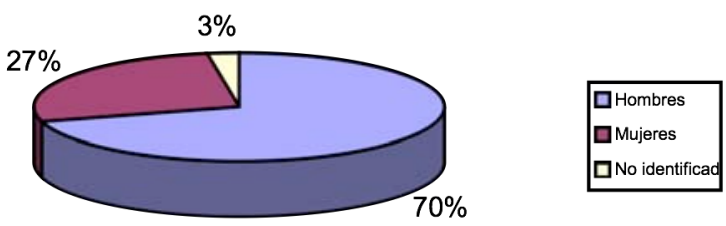

Figura 2. Género de los autores del INTA

En la última memoria anual publicada por el INTA, se registran para el año 2005 un total de 380 personas calificadas de "investigadores", siendo 78 las mujeres que específicamente se dedican a I+D, con un porcentaje del $20 \%$. Por lo tanto, el porcentaje desciende de la media nacional. $Y$ tampoco es que el porcentaje de productividad sea muy elevado, ya que sólo un $26 \%$ de las mismas han firmado alguna de las contribuciones recogidas. 


\subsection{Clasificación temática}

La clasificación en grandes áreas y, dentro de ellas, en disciplinas, se ha realizado siguiendo los criterios temáticos ofrecidos por el ISI en todas sus bases de datos. Hemos seleccionado este criterio, a pesar de que se han recogido 15 referencias que no incluían datos en el campo "Subject (materias)".

\subsubsection{Datos totales de las áreas temáticas}

Siguiendo la clasificación en áreas y disciplinas de las bases de datos del ISI, las referencias analizadas quedarían adscritas a las siguientes grandes categorías: Agricultura, Biología y Medio Ambiente; Biomedicina; Ciencias Sociales; Física; Ingeniería, Tecnología; Multidisciplinar; Química (Tabla 4).

Las dos áreas temáticas en la que se registran más trabajos son "Ingeniería" y "Física", aunque con datos bastante parecidos. La distancia en número de contribuciones al resto de las temáticas es muy destacada, no superando, salvo en el área de "Química", las 10 referencias (Figura 3).

\begin{tabular}{lrrr}
\hline Categorías temáticas & Total & Hombres & Mujeres \\
\hline $\begin{array}{l}\text { Agricultura, Biología y } \\
\text { Medio Ambiente }\end{array}$ & 9 & 7 & 2 \\
$\begin{array}{l}\text { Bibliotecología y C. } \\
\text { de la Información }\end{array}$ & 1 & 1 & 0 \\
Biomedicina & 5 & 5 & 0 \\
Física & 116 & 91 & 25 \\
Ingeniería, & 149 & 99 & 50 \\
Tecnología & & & \\
Multidisciplinar & 3 & 3 & 0 \\
Química & 18 & 15 & 3 \\
\hline
\end{tabular}

Tabla IV. Clasificación por categorías temáticas (datos globales).

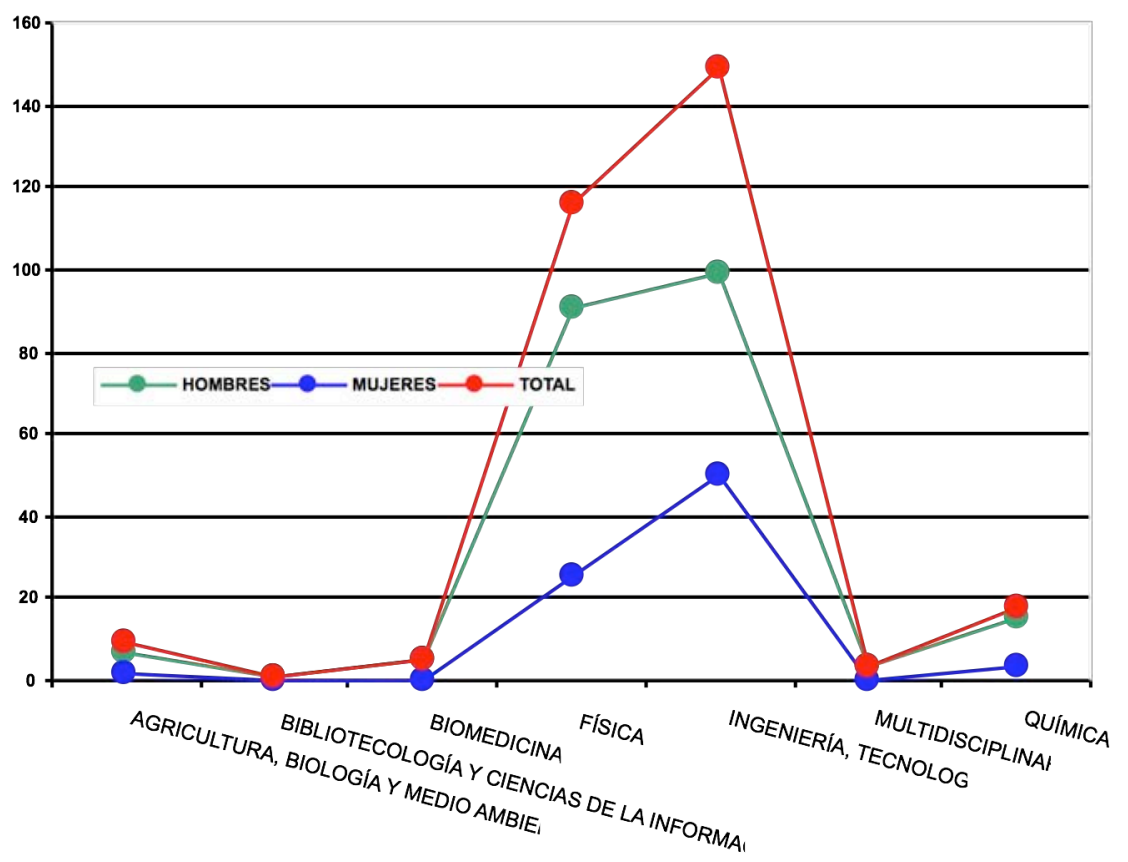

Figura 3. Distribución por sexos y por categorías temáticas

Aislando los resultados a un nivel de análisis independiente, obtenemos el mismo balance: la mayor parte de los trabajos recopilados se publican en las categorías "ingeniería" y "Física". En el caso de los hombres, la diferencia entre las dos áreas más productivas no es excesivamente destacable, algo que sí sucede en el caso de las mujeres. Otra cuestión destacable es que sólo en el caso de los hombres se registran valores en todas las categorías (Tabla 5, en apéndice I).
Las disciplinas más productivas a nivel global en el caso de los artículos son:

- Física. Meteorología y Ciencia Atmosférica: 25 referencias.

- Física. Geociencias, Multidisciplinar: 17 referencias.

- Física. Astronomía y Astrofísica: 16 referencias. 
- Ingeniería, Tecnología. Ciencias Materiales, Multidisciplinar: 14 referencias.

- Ingeniería, Tecnología. Ingeniería Aeroespacial: 8 referencias.

- Ingeniería, Tecnología. Instrumentación: 7 referencias.

- Física. Geoquímica y Geofísica: 6 referencias

- Química. Polímeros: 6 referencias.

En lo que se refiere a las mujeres, las dos disciplinas que recopilan más referencias son "Meteorología y Ciencia Atmosférica" (5 referencias) y "Geociencias, Multidisciplinar" (3 referencias). Los hombres generan más contribuciones en las áreas de "Meteorología y Ciencia Atmosférica" (20 referencias), "Astronomía y Astrofísica" (15 referencias) y "Geociencias, Multidisciplinar" (14 referencias). Las disciplinas más productivas a nivel global en el caso de las ponencias son (Tabla 6, en apéndice I):

- Física, Astronomía y Astrofísica: 12 referencias.

- Ingeniería, Tecnología. Materiales, Multidisciplinar: 13 referencias.

- Ingeniería, Tecnología. Ingeniería Aeroespacial: 19 referencias.

- Ingeniería, Tecnología. Instrumentación: 13 referencias.

- Ingeniería, Tecnología. Ingeniería Eléctrica y Electrónica: 9 referencias.

- Ingeniería, Tecnología. Óptica: 6 referencias.

- Ingeniería, Tecnología. Energía y Combustibles: 7 referencias.

En lo que se refiere a las mujeres, las dos disciplinas que recopilan más referencias son "Ingeniería Aeroespacial" (9 referencias) y "Materiales, Multidisciplinar" (9 referencias). Los hombres generan más contribuciones en las áreas de "Ingeniería Aeroespacial" (10 referencias) e "Instrumentación" (10 referencias). Sería, por lo tanto, la disciplina etiquetada como "Ingeniería Aeroespacial" la disciplina a la que más se dedican ambos géneros por separado en este tipo de contribuciones. Las disciplinas más productivas a nivel global en el caso de las ponencias son (Tabla 7 , en apéndice I):

- Física. Astronomía y Astrofísica: 28 referencias.

- Ingeniería, Tecnología. Ingeniería Aeroespacial: 27 referencias.

- Física. Meteorología y Ciencia Atmosférica: 27 referencias.
- Ingeniería, Tecnología. Materiales, Multidisciplinar: 27 referencias.

- Física. Geociencias, Multidisciplinar: 22 referencias.

- Ingeniería, Tecnología. Instrumentación: 20 referencias.

- Ingeniería, Tecnología. Ingeniería Eléctrica y Electrónica: 13 referencias.

- Ingeniería, Tecnología. Óptica: 9 referencias.

- Ingeniería, Tecnología. Energía y Combustibles: 9 referencias.

- Química. Polímeros: 7 referencias.

Las mujeres registran un mayor número de contribuciones en las disciplinas de "Ingeniería Aeroespacial" (10 referencias) y a "Materiales, Multidisciplinar" (16 referencias), mientras que sus colegas masculinos se dedican preferentemente a la "Astronomía y Astrofísica" (21 referencias), a la "Meteorología y Ciencia Atmosférica" (20 referencias), a la "Geociencias, Multidisciplinar" (17 referencias) y a la "Ingeniería Aeroespacial" (16 referencias).

\section{Conclusiones}

1. El número total de contribuciones firmadas por las investigadoras del INTA es 82.

2. Se decantan preferentemente por la publicación en artículos de revistas frente a ponencias u otro tipo de géneros documentales.

3. Su media de contribuciones anual se sitúa en 5.

4. El año en el que se registra un mayor número de contribuciones es el año 2000 para las mujeres; el 2002, para los hombres.

5. No se registra ninguna contribución en los años 1991-1992.

6. Las categorías más productivas a nivel global son la "Ingeniería, Tecnología" y la "Física", tanto para hombres como para mujeres.

7. La disciplina más productiva a nivel global es "Astronomía y Astrofísica".

8. Las mujeres se dedican en mayor número a la "Ingeniería Aeroespacial" y a los "Materiales, Multidisciplinar".

\section{Referencias}

Braisher, Tamsin L.; Symonds, Matthew R. E.; Gemmell, Neil J. (2005). Publication success in Nature and Science is not gender dependent. // BioEssays. 27 (2005) 858-859. 
Cronin, Blaise; Davenport, Elisabeth; Martison, Anna (1997) Women's Studies: Bibliometric and content analysis of the formative years. // Journal of Documentation. 53:2 (March 1997) 123-138.

Fuchs, Stefan; Stebut, Janina von; Allmendinger, Jutta (2001). Gender, Science, and Scientific Organizations in Germany. // Minerva. 39 (2001) 175-201.

Kretschmer, Hildrum; Aguillo, Isidro F. (2005). New indicators for gender studies in Web networks. // Information Processing and Management. 41 (2005) 1481-1494.

Leta, Jacqueline; Lewison, Grant (2003). The contribution of women in Brazilian science: A case study in astronomy, immunology and oceanography. // Scientometrics. 53:3 (2003) 339-353.

Licea de Arenas, Judith; Sandoval, Martín; Arenas, Miguel (2003). La investigación agrícola en México: Un estudio bibliométrico con enfoque de género. // Anales de Documentación. 6 (2003) 145-154.

Mauleón, Elba; Bordons, María (2006). Productivity, impact and publication habits by gender in the area of Materials Science. // Scientometrics. 66:1 (2006) 199-218.

Prpic, Katarina (2002). Gender and productivity differentials in science. // Scientometrics. 55:1 (2002) 27-58.

\section{Apéndice I}

\begin{tabular}{|c|c|c|c|c|}
\hline Categorías & Disciplinas & INTA & Hombres & Mujeres \\
\hline Agricultura, Biología y Medio Ambiente & Medio Ambiente & 3 & 2 & 1 \\
\hline Agricultura, Biología y Medio Ambiente & Biotecn. y Microbiol. Aplic. & 2 & 2 & 0 \\
\hline Biomedicina & Bioquímica y Biología Molecular & 2 & 2 & 0 \\
\hline Biomedicina & Biofísica & 2 & 2 & 0 \\
\hline Ciencias Sociales & Bibliotec. y Ciencias de la Inf. & 1 & 1 & 0 \\
\hline Física & $\begin{array}{l}\text { Meteorología y } \quad \text { Ciencia } \\
\text { Atmosférica }\end{array}$ & 25 & 20 & 5 \\
\hline Física & Astronomía y Astrofísica & 16 & 15 & 1 \\
\hline Física & Geociencias, Multidisciplinar & 17 & 14 & 3 \\
\hline Física & Geoquímica y Geofísica & 6 & 6 & 0 \\
\hline Fisica & Física Aplicada & 9 & 8 & 1 \\
\hline Física & Termodinámica & 4 & 4 & 0 \\
\hline Física & Física, Estado Sólido & 4 & 3 & 1 \\
\hline Ingeniería, Tecnología & Materiales, Multidisciplinar & 14 & 7 & 7 \\
\hline Ingeniería, Tecnología & Ingeniería Aeroespacial & 8 & 7 & 1 \\
\hline Ingeniería, Tecnología & Instrumentación & 7 & 6 & 1 \\
\hline Ingeniería, Tecnología & Materiales, Revest. y Películas & 4 & 3 & 1 \\
\hline Ingeniería, Tecnología & Ingeniería Eléctric. y Electrón. & 4 & 4 & 0 \\
\hline Ingeniería, Tecnología & Metalurgia e Ing. Metalúrgica & 4 & 1 & 3 \\
\hline Ingeniería, Tecnología & Ingeniería Mecánica & 3 & 3 & 0 \\
\hline Ingeniería, Tecnología & Óptica & 3 & 3 & 0 \\
\hline Ingeniería, Tecnología & Energía y Combustibles & 2 & 2 & 0 \\
\hline Ingeniería, Tecnología & Ingeniería Química & 2 & 2 & 0 \\
\hline Ingeniería, Tecnología & Ingeniería, Multidisciplinar & 2 & 2 & 0 \\
\hline Ingeniería, Tecnología & Mecánica & 2 & 2 & 0 \\
\hline Ingeniería, Tecnología & Nanociencia y Nanotecnología & 2 & 2 & 0 \\
\hline Ingeniería, Tecnología & Materiales, Cerámica & 2 & 1 & 1 \\
\hline Ingeniería, Tecnología & Acústica & 1 & 1 & 0 \\
\hline Ingeniería, Tecnología & Informática, Aplic. multidisc. & 1 & 1 & 0 \\
\hline Ingeniería, Tecnología & Materiales, Mater. Compuestos & 1 & 1 & 0 \\
\hline Ingeniería, Tecnología & Ingeniería Industrial & 1 & 0 & 1 \\
\hline Ingeniería, Tecnología & Ingeniería de Fabricación & 1 & 0 & 1 \\
\hline Multidisciplinar & Ciencias Multidisciplinares & 3 & 3 & 0 \\
\hline Química & Polímeros & 6 & 6 & 0 \\
\hline Química & Química, Multidisciplinar & 3 & 2 & 1 \\
\hline Química & Bioquímica & 2 & 2 & 0 \\
\hline
\end{tabular}

Tabla V. Clasificación por materias (Artículos). enfoque de género en el período 1990-2005. Estudio de un caso concreto. // Ibersid. (2007) 379-386. ISSN 1888-0967. 


\begin{tabular}{|c|c|c|c|c|}
\hline Categorías & Disciplinas & INTA & Hombres & Mujeres \\
\hline $\begin{array}{l}\text { Agricultura, Biología y Medio } \\
\text { Ambiente }\end{array}$ & Medio Ambiente & 2 & 1 & 1 \\
\hline $\begin{array}{l}\text { Agricultura, Biología y Medio } \\
\text { Ambiente }\end{array}$ & Biotecn. y Microbiol. Aplic. & 2 & 2 & 0 \\
\hline Biomedicina & Genética y Herencia & 1 & 1 & 0 \\
\hline Física & Meteorología y Ciencia Atmosférica & 2 & 0 & 2 \\
\hline Física & Astronomía y Astrofísica & 12 & 6 & 6 \\
\hline Física & Geociencias, Multidisciplinar & 5 & 3 & 2 \\
\hline Física & Geoquímica y Geofísica & 1 & 1 & 0 \\
\hline Fisica & Física Aplicada & 6 & 3 & 3 \\
\hline Física & Termodinámica & 2 & 2 & 0 \\
\hline Física & Física, Materiales condensados & 4 & 2 & 2 \\
\hline Física & Espectrocopia & 3 & 2 & 1 \\
\hline Ingeniería, Tecnología & Ingeniería Aeroespacial & 19 & 10 & 9 \\
\hline Ingeniería, Tecnología & Materiales, Multidisciplinar & 13 & 4 & 9 \\
\hline Ingeniería, Tecnología & Instrumentación & 13 & 10 & 3 \\
\hline Ingeniería, Tecnología & Ingeniería Mecánica & 5 & 4 & 1 \\
\hline Ingeniería, Tecnología & Materiales, Revest. y Películas & 4 & 1 & 3 \\
\hline Ingeniería, Tecnología & Ingeniería Eléctric. y Electrón. & 9 & 7 & 2 \\
\hline Ingeniería, Tecnología & Óptica & 6 & 5 & 1 \\
\hline Ingeniería, Tecnología & Energía y Combustibles & 7 & 5 & 2 \\
\hline Ingeniería, Tecnología & Ingeniería Química & 2 & 2 & 0 \\
\hline Ingeniería, Tecnología & Mecánica & 2 & 1 & 1 \\
\hline Ingeniería, Tecnología & Informática, Aplic. multidisc. & 1 & 1 & 0 \\
\hline Ingeniería, Tecnología & Materiales, Cerámica & 1 & 0 & 1 \\
\hline Ingeniería, Tecnología & Informática, Hardware & 1 & 1 & 0 \\
\hline Ingeniería, Tecnología & Informática, Software & 1 & 1 & 0 \\
\hline Ingeniería, Tecnología & Ingeniería Industrial & 1 & 0 & 1 \\
\hline Ingeniería, Tecnología & Informática, Apl. Interdisciplinares & 1 & 0 & 1 \\
\hline Ingeniería, Tecnología & Ingeniería de Fabricación & 1 & 0 & 1 \\
\hline Ingeniería, Tecnología & $\begin{array}{l}\text { Ciencias de los materiales, caracterización y } \\
\text { prueba }\end{array}$ & 1 & 1 & 0 \\
\hline Ingeniería, Tecnología & Nanociencia y Nanotecnología & 1 & 0 & 1 \\
\hline Química & Química aplicada & 3 & 2 & 1 \\
\hline Química & Química orgánica & 3 & 2 & 1 \\
\hline Química & Polímeros & 1 & 1 & 0 \\
\hline
\end{tabular}

Tabla VI. Clasificación por materias (Ponencias). enfoque de género en el período 1990-2005. Estudio de un caso concreto. // Ibersid. (2007) 379-386. ISSN 1888-0967. 


\begin{tabular}{|c|c|c|c|c|}
\hline Categorías & Disciplinas & INTA & Hombres & Mujeres \\
\hline Agricultura, Biología y Medio Ambiente & Medio Ambiente & 5 & 3 & 2 \\
\hline Agricultura, Biología y Medio Ambiente & Biotecn. y Microbiol. Aplic. & 4 & 4 & 0 \\
\hline Biomedicina & Bioquímica y Biología Molecular & 2 & 2 & 0 \\
\hline Biomedicina & Biofísica & 2 & 2 & 0 \\
\hline Biomedicina & Genética y Herencia & 1 & 1 & 0 \\
\hline Ciencias Sociales & Bibliotecología y Ciencias de la información & 1 & 1 & 0 \\
\hline Física & Meteorología y Ciencia Atmosférica & 27 & 20 & 7 \\
\hline Física & Astronomía y Astrofísica & 28 & 21 & 7 \\
\hline Física & Geociencias, Multidisciplinar & 22 & 17 & 5 \\
\hline Física & Geoquímica y Geofísica & 7 & 7 & 0 \\
\hline Fisica & Física Aplicada & 15 & 11 & 4 \\
\hline Física & Termodinámica & 6 & 6 & 0 \\
\hline Física & Física, Materiales condensados & 5 & 2 & 3 \\
\hline Física & Nanociencia y Nanotecnología & 3 & 2 & 1 \\
\hline Física & Espectrocopia & 3 & 2 & 1 \\
\hline Física & Física, Estado Sólido & 3 & 3 & 0 \\
\hline Ingeniería, Tecnología & Ingeniería Aeroespacial & 27 & 17 & 10 \\
\hline Ingeniería, Tecnología & Materiales, Multidisciplinar & 27 & 11 & 16 \\
\hline Ingeniería, Tecnología & Instrumentación & 20 & 16 & 4 \\
\hline Ingeniería, Tecnología & Ingeniería Mecánica & 8 & 7 & 1 \\
\hline Ingeniería, Tecnología & Materiales, Revest. y Películas & 8 & 4 & 4 \\
\hline Ingeniería, Tecnología & Ingeniería Eléctric. y Electrón. & 13 & 11 & 2 \\
\hline Ingeniería, Tecnología & Óptica & 9 & 8 & 1 \\
\hline Ingeniería, Tecnología & Energía y Combustibles & 9 & 7 & 2 \\
\hline Ingeniería, Tecnología & Metalurgia e Ing. Metalúrgica & 4 & 1 & 3 \\
\hline Ingeniería, Tecnología & Ingeniería Química & 4 & 4 & 0 \\
\hline Ingeniería, Tecnología & Ingeniería, Multidisciplinar & 2 & 2 & 0 \\
\hline Ingeniería, Tecnología & Mecánica & 4 & 3 & 1 \\
\hline Ingeniería, Tecnología & Acústica & 1 & 1 & 0 \\
\hline Ingeniería, Tecnología & Informática, Aplic. multidisc. & 2 & 2 & 0 \\
\hline Ingeniería, Tecnología & Materiales, Cerámica & 3 & 1 & 2 \\
\hline Ingeniería, Tecnología & Materiales, Mater. Compuestos & 1 & 1 & 0 \\
\hline Ingeniería, Tecnología & Informática, Hardware & 1 & 1 & 0 \\
\hline Ingeniería, Tecnología & Informática, Software & 1 & 1 & 0 \\
\hline Ingeniería, Tecnología & Ingeniería Industrial & 2 & 0 & 2 \\
\hline Ingeniería, Tecnología & Informática, Apl. Interdisciplinares & 1 & 0 & 1 \\
\hline Ingeniería, Tecnología & Ingeniería de Fabricación & 1 & 0 & 1 \\
\hline Ingeniería, Tecnología & Materiales, caract. y prueba & 1 & 1 & 0 \\
\hline Multidisciplinar & Ciencias Multidisciplinares & 3 & 3 & 0 \\
\hline Química & Polímeros & 7 & 7 & 0 \\
\hline Química & Química, Multidisciplinar & 3 & 2 & 1 \\
\hline Química & Bioquímica & 2 & 2 & 0 \\
\hline Química & Química aplicada & 3 & 2 & 1 \\
\hline Química & Química orgánica & 3 & 2 & 1 \\
\hline
\end{tabular}

Tabla VII. Clasificación por categorías temáticas y por disciplinas (datos globales)

Paz, Silvia; Herrerías, Rocio; Mérida, Fernando. Análisis de la producción científica española en investigación aeronáutica con un enfoque de género en el período 1990-2005. Estudio de un caso concreto. // Ibersid. (2007) 379-386. ISSN 1888-0967. 